\title{
Problems in Pronunciation and Perception of Mandarin Tones: An Empirical Study on Bangladeshi Undergraduate Students in China
}

\author{
Soniya Akter \\ Nantong University \\ Jianqing Song \\ Nantong University
}

\begin{abstract}
Tone is a vital part of Mandarin. This study will explore the problems in pronunciation and perception of Mandarin tone for Bangladeshi undergraduate students in China. Both quantitative and qualitative approaches have been engaged to collect the necessary information from the randomly selected one hundred students of the three selected Universities. From the study, several factors have been found to be barriers in the way of learning Mandarin. It is important to motivate MFL learners to realize the necessity to tone learning as some learners are indifferent to tone learning. This study presents several recommendations for Mandarin learners, which will be helpful for the learners.
\end{abstract}

Keywords: Bengali MFL learners, Mandarin tones, non-tonal background, pronunciation, problems

\section{INTRODUCTION}

There is an issue in perception and pronunciation of Mandarin Tone for foreign learners of Mandarin language, particularly for Bengali learners because Bengali learner's native language is Bangla which is a non-tonal language, for this the Bengali learners cannot maintain tone level and if they try to focus on tones then their learning becomes hindered. This is the reason of their ignoring the tones while learning Mandarin. Beginner level Mandarin learners get confused with four tones. There are four tones in Mandarin language and a neutral tone as well, but the neutral tone is not a big problem for learners so this study only focuses on four tones. Those four tones make the learners disappointed and repulse their learning interest. This study focuses on the tone related problem of Bengali learners. Tonal problem is a major obstacle to learning Mandarin as foreign language (MFL). The purpose of this study is to measure the difficulty of undergraduate Bangladeshi students' specific problem in perception and pronunciation of Mandarin tones. Mandarin is a tonal language and every syllable has its radical static tone, where both high-low disparities and rising-falling alternation are situated which has great importance in conveying meaning in words but beginner level learners are unmindful of that importance.

\section{Problem Statement}

Bangladeshi learners are from non-tonal language background so, they do not know the importance of tone in a language, for that they are indifferent of tone learning. As they are new with the term of "Tone" 
so, most of them show negligence with this study. Another thing is that, interest is a big factor for learning anything, but as they are indifferent with tone learning that is why they fail to pronounce and identify tones.

\section{Objectives}

The objective of this study is to find out the specific problems in pronunciation and perception in Mandarin tones and make a spectrum of the most difficult tones for Bengali MFL learners. This study has focused on attaining the following objectives:

a. To present the problems that they feel to learn Mandarin tones.

b. To present the most difficult Mandarin tones to pronounce for Bengali MFL learners.

c. To present the most difficult Mandarin tones to perceive for Bengali MFL learners.

d. To present the overall correction and error rate for both pronunciation and perception.

e. To prepare suggestions or recommendations for future researchers.

\section{Research Questions}

On the basis of above research objectives, this study plans to seek answer to the following questions:

a. What are the major problems Bangladeshi beginner level learners feel in learning to pronounce and perceive the Mandarin tone?

b. What are the problems they face in learning Mandarin tone?

This study has attempted to determine the answer to the above questions.

\section{LITERATURE REVIEW}

Tones are very important for tonal language. Mandarin or Chinese language is a tonal language so it is pretty important to focus on the tone level to learn Mandarin. Each single Chinese character has one tone. There are four tones in mandarin and a neutral tone as well. A same syllable with a different tone presents different meaning. Each syllable of Mandarin has its static tone. The falling and rising, high and low pitches depend on the frequency of a vibrancy of the vocal cords. Mandarin tone formation is indeterminate; one can pass not only the tone level, but also the transition mode. There are levels, going up, going down going up and going down. According to Hiroshi (2003), "There are two types of accents in the languages of the world. The first one is 'stress accent'; it uses the intensity of sounds to differentiate various lexical items. The second one is 'pitch accent'; it uses the pitch of sounds to distinguish one word from another." Bangla is a non-tonal language, but intonational tunes and underlying tones can exist in Bangla language. Bangla language does not use tones as contrastive feature. According to Kamrul Hasan, "Bangla does not show any overtly realized bitonal pitch accents. The notion of boundary tone implies that the pitch object is aligned with the original edge of a phrase rather than a certain syllable". In Bangla, identical tones do not occur in sequence. Bangla Language does not show any rise+flat+rise sequences. Four Mandarin tones are-

- The first tone is flat, so it is the level tone $-\overline{\mathrm{A}}$

- The second tone is a rise from the middle to high, so it is called the rising- $\mathrm{A}$

- The third tone is from low to lower and then rises, so it is called the falling-rising tone- ă

- The fourth tone is a fall from the top to the bottom, so it is called the falling tone- à

The existing research thinks that the confusion between the falling-rising tone and the rising tones are the most prominent problem for non-tonal native speakers as well as MFL learners, but this study has found the different scenario of Bengali MFL learns; and this is the difference between Bangladeshi students' perception and pronunciation of Mandarin tones than other learners.

The most effective and the easiest pathway to record tones and transcribe are the manner of tone-letter introduced by Chao (1968). Tone pitch is classified into five degrees and to mark the certain location of the tone pitch on the balance it shares a vertical line into four parts. According to Zhaojie Peng and Min Zhao (2019), "It is natural to make the pitch in the second syllable lower than that in the first". Study found that the perception and pronunciation ability of Bangladeshi students in mandarin tones gradually increases with the increase of Chinese level, especially in advanced level. Mandarin is a tonal language; the pitch in 
modern Mandarin Chinese serves a contrastive function. Tone plays a vital role in distinguishing word meanings. "The first and second word's tones are compromised for the sake of being euphonious in a Chinese disyllabic phrase" (Wu, 1992).

Pitch is used to sustain emotions and nuances. The pitch plays an important role to differentiate lexical meaning in the tonal language like Mandarin, though tonal languages even have intonation features. Here we can make this point clear by the following example, if one says in English 'Three'? With the ascending or rising pitch to ask a question, or 'Three'! There is a downing or falling pitch to impart a requirement. It is apparent that, variety in pitch carried the diverse sentence functions or emotions, though they do not switch the word's sense: 'three' is still the number 'three', though in some languages, pitch leads at the word level. Pitch is a contrastive feature in a tonal language like Chinese.

\section{METHODOLOGY}

A mixed-method has been applied for this study. A covert participant observation, semi-structured individual interviews and questionnaire survey have been involved in this study to ensure both quantitative and qualitative forms of data collection.

The quantitative data collection through covert participant observation has been arranged from September $20^{\text {th }}$ to December $20^{\text {th }}, 2019$. All the undergraduate students had Chinese language classes for 3 hours per week that continued for 4 months. The qualitative data collection through individual interviews has been conducted from October $1^{\text {st }}$ to October $6^{\text {th }}, 2019$. These interview sessions have been carried on for thirty minutes per day and two interviewees have been interviewed on each day of the sessions. Fifteen minutes have been taken for each interview to get the required information in the best possible manner. The questionnaire survey's quantitative data collection has been carried out from April $1^{\text {st }}$ to April $15^{\text {th }}, 2020$. This research has been established as valid since the objectives have been brought by providing all the necessary materials or items, that the study intends to measure. This research is reliable since the methodology has been assured of producing the same, stable, and consistent results while being conducted in the same context with the same participants using different research instruments.

\section{Participants}

Twenty-five Bangladeshi Undergraduate Students of Nantong University (China) were the subject of this study for covert participant observation, fifteen students among this population have been selected randomly for individual interviews and randomly selected one hundred (100) students of Nanjing Tech University (China) for a questionnaire survey. All of them were from different regions of Bangladesh and their mother tongue is Bangla, aged between 18 to 30. All of them were fresh men and just started Chinese language class.

\section{Materials}

In the period of observation, all the respondents were read out the Mandarin words by following their teacher. The respondents just imitated the sound of the words and were not conscious of tones. In this period, the data has been collected by field note taking by observing the participant's responses. In the period of individual interview, the data has been collected by field note taking by the participant's responses, interactions, processes, and behaviors regarding Mandarin tone learning. Respondents were being asked about some relevant open-ended and close-ended questions, thus qualitative data and quantitative data have been collected. In a questionnaire survey, some relevant Mandarin tone related questions have been included. So, data has been collected through covert participant observation, individual interview and a questionnaire survey.

\section{Procedure}

The study was divided into three parts. The first part was to collect quantitative data by covert participant observation. All the respondents were read out the Mandarin words by following their teacher, but they just imitated the sound of the words and never conscious of tones. After that the teacher asked the 
students to listen to the words from him and write down the right answer on the answer sheet with four tones. The second part was to collect qualitative data through a semi structured interview, where the subjects were asked to read out 4 Mandarin words with different tones and asked to share their thoughts regarding Mandarin tone learning. To prevent the respondents from predicting the answers from memorization, the tones were asked to speak in random order. The third part was to collect quantitative data through a questionnaire survey, where there was a question about the most difficult mandarin tone from his/her own point of view and what kind of problem they feel in learning the Mandarin tone. Thus, this study issued the problems that they feel in learning Mandarin tones and measured the major difficulties in pronunciation and perception of Mandarin tones for Bangladeshi beginner level learners.

\section{Method of Analysis}

The data of this study has been collected by note taking from observation, individual interview, and a questionnaire survey. In the observation period, it has been noted that how many students had a problem in Mandarin tone pronunciation and perception and what kind of problems they felt to maintain the tone level. These data were collected by observing their performance in Chinese language class. It has been noted, whether they really tried to maintain tone levels or ignored them, whether they were conscious of tone importance or completely unmindful. These all have been done by using the verity of Mandarin words and tones as well. In individual interview session, subjects were asked to hear some Mandarin words from native speakers and try to imitate them; they were also asked to share their point of view regarding Mandarin tone maintenance and if they really felt it important while talking or useless and if they wanted to share anything about mandarin tone learning. In survey questionnaire section, subjects had to answer some relevant Mandarin tone related question like- what kind of problem they feel to learn Mandarin tone, which one is the most difficult to pronounce and perceive for him/her and some other relevant questions as well.

\section{RESULTS AND DISCUSSIONS}

A covert participant observation with a semi-structured interview and a questionnaire survey has been conducted to bring out data for this study to answer the study's research questions. In this section, data have been presented and delineated along with the instruments used for data collection.

In the previous section of the methodological approach, it has been mentioned that, the researchers carried out a covert participant observation and semi-structured interviews by choosing twenty-five participants and ten interviewees and respectively from the same study population of 25 students of the specific university for the quantitative and the qualitative study. The study results have been cross checked with the results of the individual questions used for the questionnaire survey. It has been brought to light by a covert participant observation that none of them learned Mandarin tone for behaving like native Chinese speakers. Furthermore, many of them were careless about tones, and some of them did not know the importance of tones and others failed to understand and maintain the tone level. Through the semistructured interview, it has been found that most of the participants claimed that they are highly interested to learn Mandarin tone as a native speaker, but they felt troublesome to pronounce and perceive mandarin tones and most of the time they got confused with the tones and failed to differentiate from one to another.

\section{Results From the Quantitative Study (Questionnaire Survey \& Covert Participants Observation)}

The data from quantitative study is collected by questionnaire survey and covert participants observation. Data of quantitative study is presented through a table here. 
TABLE 1

RESULTS OF QUESTIONNAIRE SURVEY

\begin{tabular}{|c|c|c|c|c|c|}
\hline \multicolumn{2}{|c|}{ Major problems of learning tones } & \multicolumn{2}{|c|}{ Status of leaning Interest } & \multicolumn{2}{c|}{$\begin{array}{c}\text { Importance of tones to the } \\
\text { learners }\end{array}$} \\
\hline Major problems & Result & Interest level & Result & $\begin{array}{c}\text { Importance } \\
\text { level }\end{array}$ & Result \\
\hline Difficult to learn & $28.75 \%$ & Highly Interested & $41.75 \%$ & Yes & $29.75 \%$ \\
\hline $\begin{array}{c}\text { Confusing to } \\
\text { understand }\end{array}$ & $37.25 \%$ & Interested & $29.25 \%$ & No & $18.52 \%$ \\
\hline $\begin{array}{c}\text { Unmindful of tone's } \\
\text { importance }\end{array}$ & $21.52 \%$ & Not Interested & $17.54 \%$ & Maybe & $38.54 \%$ \\
\hline Other & $12.48 \%$ & Neutral & $11.46 \%$ & No comment & $13.19 \%$ \\
\hline
\end{tabular}

The data of the questionnaire survey have been presented in Table 1. It has been established that the followings are the major factors that influence the learning Mandarin tones and these problems hinder the MFL learner's Mandarin tone learning, where most of the learners are highly interested to learn Mandarin but most of the learners do not know the importance of tones in the language (See Table 1). This study found that, the majority of the learners get confused to differentiate the four tones. Another problem is that, most of them feel it difficult to learn because they are not well familiar with the concept of the "Tone" as they are from the non-tonal language background. Some learners stated that they are unmindful of mandarin tone's importance in learning Mandarin language and there are some other reasons as well.

From the questionnaire survey it is found that, most of the participants agreed about the basic problem about the tone learning that is, they are from the non-tonal language background so they are careless of tone learning and its importance but still they are highly interested in Mandarin learning. The students were not able to achieve the perfect tone pronunciation and perception. Besides, often they cannot differentiate the four tones at all. Since it does not exist in the Bangla language, it may lead to the confusion that the nonnative speakers of Mandarin often mistakenly skip the tonal part of Mandarin language. We find that the learner's confusion level of Mandarin tones understanding reach the top $(37.25 \%)$ in the table among the four points, that learners feel it difficult to learn is placed in the second position $(28.75 \%)$ in the table, some learners are fully unmindful of its importance $(21.52 \%)$ and they do not try to maintain tone levels at all. Hence, there are some other reasons (12.48\%) that hinder the Mandarin tone learning (See table 1). These are the major issues that become the barriers in the way of learning Mandarin tone for Bangladeshi students.

Furthermore, most of the Bengali MFL learners are highly interested (41.75\%) in Mandarin learning, though some of them are not interested (17.54\%) at all. According to the statistical table 1, it is obvious that, the "Interested" numbers of students are much more $(41.75 \%>17.54)$ than the "Not Interested" number of students.

Besides, most of the students do not know about the importance of tones in learning a language. The number of students of "Having knowledge" (29.75\%) of its importance is lower than "Not having knowledge/ unmindful of its importance" (38.54\%). Hence, some of them are thinking that tones are not important $(18.52 \%)$ in learning a language.

With the above-mentioned elaboration, there are some other issues too. Other issues will be presented in Table 2 combining with the collected data from the covert participant observation. 
TABLE 2

STATISTICAL TABLE OF THE CORRECTION AND ERROR RATE OF MANDARIN TONE PRONUNCIATION AND PERCEPTION RATE FOR BENGALI MFL LEARNERS

\begin{tabular}{|c|c|c|c|c|c|c|}
\hline & \multicolumn{2}{|c|}{$\begin{array}{c}\text { Error \& correction rate } \\
\text { in pronunciation }\end{array}$} & \multicolumn{2}{|c|}{$\begin{array}{c}\text { Error \& correction rate } \\
\text { in perception }\end{array}$} & $\begin{array}{c}\text { Overall error \& correction } \\
\text { rate of pronunciation and } \\
\text { perception }\end{array}$ \\
\cline { 2 - 7 } Mandarin tones & Error rate & $\begin{array}{c}\text { Correction } \\
\text { rate }\end{array}$ & Error rate & $\begin{array}{c}\text { Correction } \\
\text { rate }\end{array}$ & $\begin{array}{c}\text { Overall } \\
\text { Overall } \\
\text { error rate }\end{array}$ & $\begin{array}{c}\text { correction } \\
\text { rate }\end{array}$ \\
\hline First tone & $61.11 \%$ & $38.89 \%$ & $77.88 \%$ & $22.12 \%$ & $69.49 \%$ & $30.50 \%$ \\
\hline Second tone & $78.23 \%$ & $21.77 \%$ & $86.46 \%$ & $13.54 \%$ & $82.34 \%$ & $17.69 \%$ \\
\hline Third tone & $68.45 \%$ & $31.55 \%$ & $65.38 \%$ & $34.62 \%$ & $66.91 \%$ & $33.08 \%$ \\
\hline Fourth tone & $82.97 \%$ & $17.03 \%$ & $88.39 \%$ & $11.61 \%$ & $85.68 \%$ & $14.32 \%$ \\
\hline Total result & $72.69 \%$ & $27.31 \%$ & $79.52 \%$ & $20.47 \%$ & $76.10 \%$ & $23.89 \%$ \\
\hline
\end{tabular}

Here it is obvious that, the Bengali MFL learners have committed the highest number of errors in pronouncing the fourth tones $(82.97 \%)$ and the second $(78.23 \%)$ tone. The percentages of errors in pronouncing and perceiving the second and the fourth tones are higher than first and third tones. So, it can be said that, the main problematic tones are the second and the fourth tones for Bangladeshi students. Figure 1 will make the above-mentioned statement clearer by portraying the overall picture in a single frame.

FIGURE 1

THE MOST DIFFICULT MANDARIN TONE FOR PRONOUNCING AND PERCEIVING ACCORDING TO THE RESPONDENTS OF BANGLADESHI UNDERGRADUATE STUDENTS

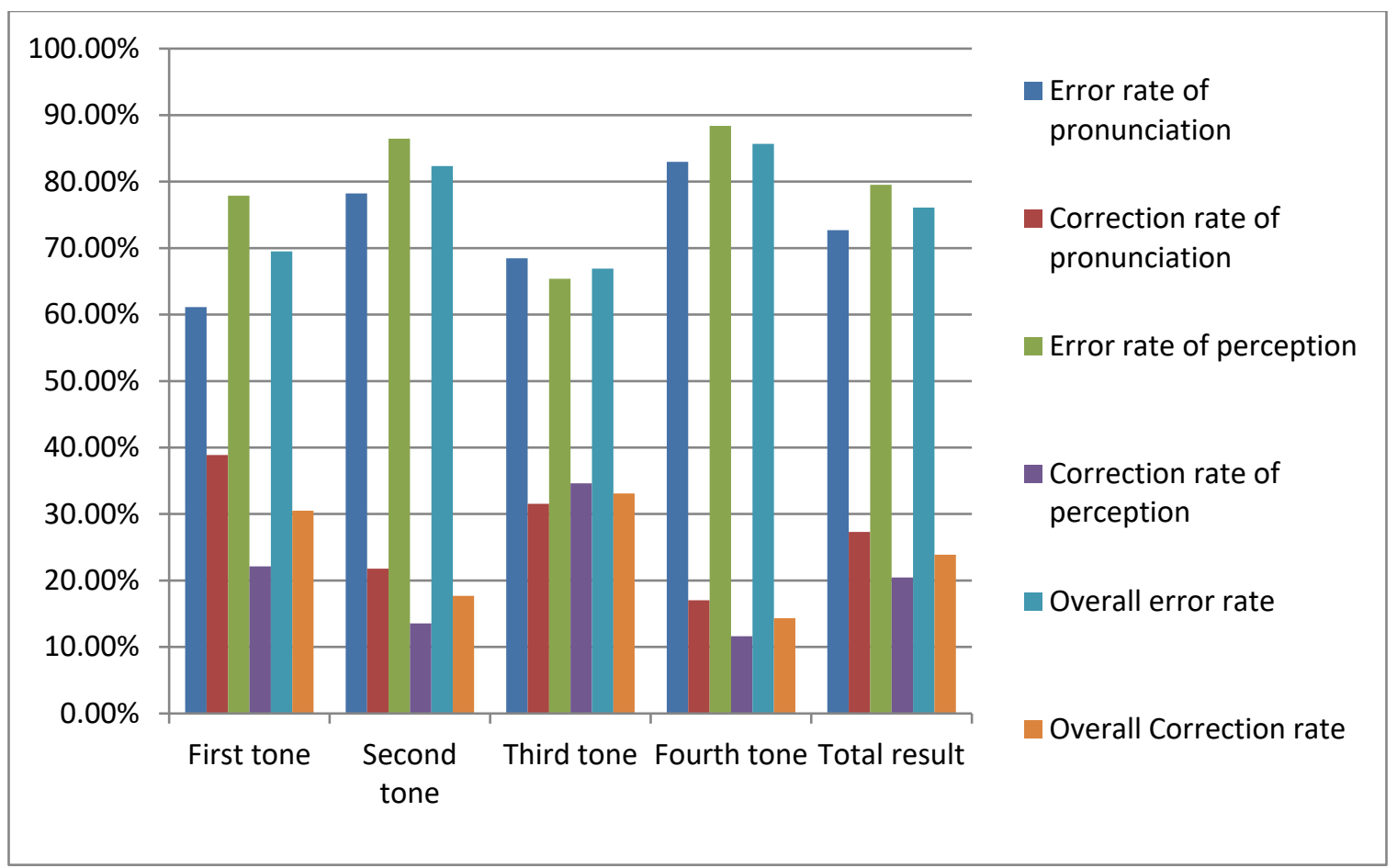

Figure 1 is represented by Table 2 . It is apparent that, the error rate of the second $(82.34 \%)$ and the fourth $(85.68 \%)$ tones are pretty higher than the first $(69.49 \%)$ and the third $(66.91 \%)$ tones for both 
pronunciation and perception, but the error rate of the fourth tone (88.39\%) in terms of the perception level is supreme in the chart.

The pronunciation and the perception rate are different from each other. Where the error rate of the first tone pronunciation is lower $(61.11 \%<77.88 \%)$ than the first tone perception, and the correction rate of the first tone pronunciation is higher $(38.89 \%>22.12 \%)$ than the first tone perception level. The error rate of the second tone pronunciation is lower $(78.23 \%<86.46 \%)$ than the second tone perception but the correction rate of the second tone pronunciation is higher $(21.77 \%>13.54 \%)$ than the second tone perception level. For the third tone, the result is become little bit different. Where, the error rate of the third tone pronunciation is higher $(68.45 \%>65.38 \%)$ than the third tone perception and the correction rate of the third tone pronunciation is lower $(31.55 \%<34.62 \%)$ than the third tone perception level. In the fourth tone, the error rate of the fourth tone pronunciation is lower $(82.97 \%<88.39 \%)$ than the fourth tone perception and the correction rate for tone pronunciation is higher $(17.03 \%>11.61 \%)$ than the fourth tone perception level. (See Table 2)

In addition, the error rates of the perception are higher $(79.52 \%>72.69 \%)$ than the pronunciation rate. Besides, the correction rate of pronunciation $(27.31 \%>20.47 \%)$ is comparatively higher than the perception rate. Furthermore, when they try to understand it from native or other speakers, they cannot perceive the tone at all. This study found that, learners get confused between the second and the fourth tones. According to the learners' opinion, they need more time for the second and the fourth tones than the first and the third tones to differentiate which one is the second tone and which one is the fourth tone when they hear from others. Here it is prominent that, the total error rate is higher $(76.10 \%>23.89 \%)$ than the total correction rate for both pronunciation and perception.

The correction rate of the third tone is the highest position in the chart for both pronunciation and perception, the first tone is in the second position and then the second and the fourth tone placed respectively in the chart. The error rate of the fourth tone has taken the highest position in the chart, where the second tone has taken the second position, then the first and the third tones are placed in the third and fourth position in the chart respectively. So, it is obvious that the second and the fourth tones are the most difficult for both pronouncing and perceiving for Bangladeshi beginner level learners (Follow Figure 1).

So, it is found that, the students faced the problem in pronouncing and perceiving the fourth tone, which has reached $85.68 \%$ of total errors. This can be an evidence of the great challenge in Mandarin tone mastery, which they usually find the most difficult and confusing. The second highest number of errors is the second tone, which is $82.34 \%$. This is followed by the first tone as $69.49 \%$ and the third tone as $66.91 \%$. By this picture, we get the Bengali MFL learner's Mandarin tone pronunciation and perception status. According to the total error rate of perception and pronunciation of the four tones of Mandarin, the subjects' mastery of the four tones can be known. Here, the total correction rate of tone perception and pronunciation of the participants were for the third, first, second and fourth tones were 33.08\%, 30.50\%, 17.66 and 14.32\% respectively, Therefore, for Bangladeshi students, the first tone and the third tone of Mandarin are easy to pronounce and perceive, though they do not meet the standard.

According to the beginning level learners, all the tones are complicated for foreign learners. But this study found that, the first and the third tones are comparatively easier than the second and the fourth tones to the Bangladeshi beginner level Mandarin learners. Learners get confused with the second and the fourth tones in most cases, where the wave of the third tone and a flat tune of the first tones can help them to memorize and identify them easily. But when they hear a long sentence or hear from the native speaker then they cannot identify the tone. Learners stated that, the tune of the fourth and the second tones sound similar to them, which make them confused to identify the tone.

\section{Result From the Qualitative Study (Semi-Structured Interview)}

It has been mentioned that a mixed method is used for this study. The researchers carried out a semistructured interview by choosing fifteen interviewees who are the participants from the same study population of 25 students of the selected university for covert participant observation. The study results have been cross checked with the results of the particular questions used for the questionnaire survey. Some statements have been collected regarding the Mandarin tone learning issue where most of them agreed about 
one thing that, they are quite unmindful about tone's importance in language. Negligence hinders their tone learning and it seems very difficult for them to learn tones because, often they feel confused about tones and cannot identify at all.

Through the semi-structured interview, it has been brought to light that beginner level learners feel it complicated to learn Mandarin tones and it is very confusing to them. Hence, some learners stated that, they feel puzzled about tones at the very beginning of learning but, after memorizing some vocabularies sometimes they can pronounce and perceive the tone of the familiar words. So, we get one idea that familiar word's tones are easy to pronounce or perceive for them but when it comes randomly with unknown words then they fail to pronounce and perceive.

The tones are the most challenging aspect of learning Chinese pronunciation for adult learners. The main focus of this study is to explore the most difficult Mandarin tone for pronunciation and perception for Bangladeshi students. Therefore, the main findings answer the research questions from both the methodological approaches in quantitative and qualitative studies. After studies, we found the above interesting results. The most prominent problems for Bengali MFL learners are rising tone and falling tone. We know that, the level tone and the falling-rising tones are quite different from each other in terms of tone type and tone value. However, Bangladeshi students feel it difficult to distinguish between the rising tone and the falling tone. The level tone of Mandarin is the most difficult to perceive and the falling-rising tone the easiest to perceive. Furthermore, the perception difficulty does not correspond to the pronunciation difficulty. In other words, the falling-rising tone is the most easily perceived. The rising tone and the falling tone are difficult to perceive and pronounce. This is because the tone of the rising tone and the falling tone do not rise or fall in a straight line. Therefore, the two are often difficult to be distinguished.

In the tone teaching for Bangladeshi students, attention should be paid not only to the distinction between rising tone and falling tone, but also to the distinction between level tone and falling-rising tone. It is obvious that, the majority of the respondents make mistakes in pronouncing and perceiving the second and fourth tones. They face the rigidity in mastering the right pronunciations and perception of the second and fourth tones. Although, they do better in the first tone and the third tone, but they are still weak in the first and the third tone and they could not meet the standard. They could not gain the prospective result in tone learning. Mandarin is a tonal language with the particular phonetic system as compared to their first and second languages. Every Chinese word has a fixed tone. Whenever a tone is pronounced incorrectly, the meaning of the word will be changed. It is ticklish for the learners to distinguish as well as pronounce the tones accurately. Therefore, mandarin pronunciation errors should be investigated from time to time as those errors will disappoint the learners to learn and to master the language, which can pervert the communication across the globe.

Generally, learners start learning Mandarin by memorizing vocabularies, where they do not pay any attention to learning tones or maintenance of tone level. As their native language is non-tonal so they are unmindful of its importance. But when they find that the same word carries different meanings depending on the tone, they start to give an importance in tone learning. Hence, it becomes very difficult to maintain tone while speaking, because they are not familiar with tonal language. According to learner's opinion, the second and the fourth tones are confusing. Some learners maintain tone level for some specific words what he/she knows well or able to perceived well but in other cases, he/she shows negligence of tone maintenance or learning.

\section{RECOMMENDATIONS}

This part of the study has provided some suggestions in the following two segments. Here we can provide some suggestions regarding Mandarin tone learning for Bangladeshi MFL learners. Here these suggestions have been carried out by observing the participant's performance and opinion from the interview.

1. Watching Chinese series can be helpful for Mandarin learners, Chinese drama series are interesting so learners will enjoy and learn, and they will not be bothering at all. 
2. Before going to China, students can try to communicate with native Chinese speakers to know more about their tone system.

3. Bangladeshi educational institutions may create a chance for their students to attend education affairs arranged by foreign universities that may help the learners to get in touch with foreign students which can be helpful for learning foreign language. By knowing more about foreign language can be helpful for learning new more languages.

4. Bangladeshi educational institutions may request institution authority to run collaborative programs with foreign educational institution which may motivate them to know/learn more about foreign languages seriously. By this, students can become familiar with other tonal languages which can make it (tone) easier to them. Otherwise students become scared and feel puzzle by facing such a new term suddenly. Because Bangladeshi students have no idea about tone as Bangla is a non-tonal language.

\section{Suggestions for Further Research}

This study considered tonal errors by only few participants. It is recommended that, in a future study of concerned issues much more participants should be included to get more irrefutable result that will be more acceptable and representative. The study may include senior level learners and teachers too. The study has collected data by focusing on a few subjects but such a study should be carried out in a larger context, including more universities to get more reliable and valid data. Though in this study we mentioned some suggestions but there is no authentic solution to solve those learning problems. Future researchers may carry out some reliable solution for learners to solve their learning problems. Hence, this research has more potential variables for future researchers.

\section{CONCLUSION}

This study specially focused on very beginning level Mandarin learners from Bangladesh. It has presented that; the native speakers of non-tonal languages are failed to pronounce and perceive Mandarin tones. This study has found the major problems in learning Mandarin tone and the most difficult Mandarin tone for pronunciation and perception for Bangladeshi students. As Bangladeshi students are from a nontonal language background so they need to headway and practice new areas of the brain through wealthy study of tones. This study has found that, the first and the third tones are comparatively easier than the second and the fourth tones to the Bangladeshi beginner level Mandarin learners. Learners get confused with the second and the fourth tones. By comparing the proportion of tonal errors of different tones, the second and the fourth tones are found to be more complicated than the first and the third tones for perception and pronunciation, but the error rate of pronunciation and perception is different. We found that, the learners are well capable of perceiving $(34.62 \%)$ the third tone, but their pronunciation error $(68.45 \%)$ is higher $(68.45 \%>34.62 \%)$ than the perception level. We found diverse differences between perception and pronunciation. There is an alternation between pronunciation and perception mistakes. As a background of non-tonal language, Bangladeshi students have a great problem regarding tone issues, since the functions of Mandarin tones and Bangla language's pitch accent is different. They sometimes can pronounce and perceive some tones, but in most cases, they face the great complexity in the pronunciation and perception of Mandarin tone. To overcome this complexity, they should practice more and more.

\section{ACKNOWLEDGEMENT}

This study is all about Mandarin tone Pronunciation and perception problems of the very beginning level learners from Bangladesh. The result might be varying in another study if the respondents are from senior level learner, because this study only focused on whom they are just start to learn Mandarin language. Furthermore, it is all about tone learning Since, the study focused on tone learning without applying them in words. So, the result can be different if tones are use in the words. In short, the result of the concerned issue's might be different in terms of subject's age, region, context and learner's level. 


\section{REFERENCES}

Bruce, H., \& Aditi, L. (1991). Bengali intonational phonology. Natural Language and Linguistic Theory. Retrieved from https://www.researchgate.net/publication/226169104_Bengali_intonational_phonology

Chao, Y. (1968). A Grammar of Spoken Chinese. Berkeley: University of California Press. Retrieved from https:/www.cambridge.org/core/journals/journal-of-asian-studies/article/grammar-ofspoken-chinese-by-yuen-ren-chao-berkeley-and-los-angeles-university-of-california-press-1965xxxvi-1109-preliminary-edition-paper/E6FE3915514850F43B4C4A50E2AAD366

Dong, Y., Tsubota, Y., \& Dantsuji, M. (2013). Difficulties in Perception and Pronunciation of Mandarin Chinese Disyllabic Word Tone Acquisition: A Study of Some Japanese University Students. (Paper Presentation). 27 th Pacific Asia Conference on Language, Information, and Computation, pp. 143-152. Retrieved from https://aclanthology.org/Y13-1012.pdf

Hasan, M.K. (2015). Focus Intonation in Bengali. Journal of Pan-Pacific Association of Applied Linguistics, 19(1), 63-88. Retrieved from https://files.eric.ed.gov/fulltext/EJ1085357.pdf

He, P. (1997). Studies in Basic Phonetic Instruction in Chinese to Japanese Students. Language Teaching and Linguistic Studies, 3, 49-50.

Hiroshi, W. (2003). The Theory of Japanese Stress and Phonetic Categorization. Journal of Wu Feng Institute of Technology, 11, 283-286.

Khor, G., Mah, B., \& Chow, V. (2017). Pronunciation Error in Mandarin among the Engineering Undergraduate: An analytical Analysis. International Academic Research Journal of Social Science, 3(1), 71-76. Retrieved from https://www.researchgate.net/publication/316235913

Nilu, A. (2021). Error Analysis in Chinese Initials Made by Bangladeshi Learners at Elementary Level. International Journal of Linguistics. Retrieved from https://www.researchgate.net/publication/351996365_Error_Analysis_in_Chinese_Initials_Made by_Bangladeshi_Learners_at_Elementary_Level.

Peng, Z., \& Z Zhao, M. (2019).An Experiment on Mandarin Single-character Tone Perception of Bangladeshi Students. 9th International Conference on Social Science and Education research (SSER 2019). Retrieved from https://webofproceedings.org/proceedings_series/ESSP/SSER\%202019/SSER30025.pdf

Si, C., Yunjuan, He., Ratree, Y., Yang, L.i, B., \& Chun, W.Y. (2019). Mechanisms of tone sandhi rule application by tonal and nontonal non-native speakers. Speech Communication. Retrieved from https://www.researchgate.net/publication/336900321_Mechanisms_of_Tone_Sandhi_Rule_Appli cation_by_Tonal_and_Non-tonal_Non-native_Speakers

Tsai, R. (2011-12). Teaching and learning the tones of Mandarin Chinese. Scotland's National Centre for Language, (24), 43-50. Retrieved from https://www.scilt.org.uk/portals/24/library/slr/issues/24/24_5_tsai.pdf

Wu, Z.J. (1992). Introduction to the Phonetics of Modern Chinese. Peking: Chinese Language Teaching.

\section{APPENDIX A: MAJOR PROBLEMS OF LEARNING, MOST DIFFICULT FOR PRONUNCIATION AND PERCEPTION OF MANDARIN TONE OF BENGALI MFL LEARNERS}

1. Are the tones important for learning Mandarin?
a. Yes
b. No
c. Maybe
d. No comment

2. Is it important to learn tones?
a. Yes
b. No
c. Maybe
d. No Comment 
3. How do you feel about Mandarin tone learning?
a. Difficult to learn
b. Difficult to memorize
c. Confusing to identify
d. Do not need to learn

4. Is it possible to pronounce the tones correctly?
a. Yes
b. No
c. Never
d. Maybe

5. Can you identify the tones while hearing from the native speakers?
a. Yes
b. No
c. Never
d. Sometimes

6. What about the learning interest of Mandarin tones?
a. Highly Interested
b. Interested
c. Not Interested
d. Neutral

7. Which one is major problem in learning Mandarin tones?
a. Difficult to learn
b. Confusing to understand
c. Unmindful of tone's importance/ negligence
d. Other

8. Which one is the most difficult to learn?
a. First tone
b. Second tone
c. Third tone
d. Fourth tone

9. Which tone is the most difficult to pronounce?
a. First tone
b. Second tone
c. Third tone
d. Fourth tone

10. Which tone is the most difficult to identify or perceive?
a. First tone
b. Second tone
c. Third tone
d. Fourth tone

11. Which one is comparatively easier to you?
a. Pronounce
b. Perceive
c. Both
d. None

12. Which one is comparatively difficult to you?
a. Pronounce
b. Perceive
c. Both
d. None

Date:

Signature of the Respondent

\section{APPENDIX B: STATEMENTS FOR SEMI-STRUCTURED INTERVIEW AND OBSERVATION}

1. Mandarin tones are difficult to learn

2. Mandarin tones are confusing, it's difficult to identify.

3. The first and the third tones are different than the second and the fourth tones. Flat tune of the first tone and wave tune of the third tone help me to identify easily. The second and the fourth tones are seemed to me very similar.

4. I cannot understand the difference between the second and the fourth tones.

5. I am highly interested to learn Mandarin but tones are very confusing and difficult to learn

6. I can identify the tones of my well familiar words

7. Tones are comparatively easier to pronounce than identify

8. I am highly interested to learn Mandarin but sometimes I got depressed by being failed to produce and perceive tones. 